\title{
TIME SERIES SENTINEL-1 SAR DATA FOR THE MAPPING OF AQUACULTURE PONDS IN COASTAL ASIA
}

\author{
Marco Ottinger ${ }^{1}$, Kersten Clauss ${ }^{1}$, Juliane Huth ${ }^{2}$, Christina Eisfelder ${ }^{2}$, Patrick Leinenkugel ${ }^{2}$ and \\ Claudia Kuenzer ${ }^{2}$
}
${ }^{1}$ Department of Remote Sensing, Institute of Geography and Geology, University of Wuerzburg, 97074
Wuerzburg, Germany
${ }^{2}$ German Remote Sensing Data Center (DFD), Earth Observation Center (EOC), German Aerospace Center (DLR), 82234 Wessling, Germany

\begin{abstract}
The farming of aquatic organisms, such as fish, shrimp and mollusks has experienced considerable growth during the past 30 years. In this study, we present a method for the derivation of aquaculture ponds from high resolution SAR time series data and its potential for the approximation of aquaculture harvest volume. The Copernicus Sentinel-1 mission allows continuous high resolution radar mapping, enabling the exploitation of large data volume. Open source thresholding and segmentation algorithms were applied to extract aquaculture ponds from Sentinel-1 time series data based on object and shape metrics. With the collection of statistical and in-situ data this study estimated production for the detected aquaculture areas. In the view of its growing importance for nutrition and future food security, earth observation holds great potential as a helping tool for the sustainable management of aquaculture and estimation of pond production.
\end{abstract}

Index Terms - Aquaculture, Sentinel-1, time series data, segmentation, production estimation

\section{INTRODUCTION}

Aquaculture is the fastest growing sector in the global food industry. While capture fishery revenues have stagnated since the 1990s, aquaculture's share of total production of aquatic products has increased sharply to 45 percent (see figure 1). With an average annual growth rate of $6.7 \%$, world aquaculture production increased from 13 million tonnes in 1990 to 76 million tonnes in 2015 [1]. Today every second fish comes from aquaculture. The growing global population and changing consumer behavior are important reasons for the increased demand for aquatic products: since the 1960s, annual average fish consumption per capita has almost doubled from $10 \mathrm{~kg}$ to $19 \mathrm{~kg}$. As an essential source of valuable fatty acids and protein [2], aquaculture contributes to global food security [3] and nutrition to the growing world population. Almost two-thirds of the total global aquaculture production is produced in freshwater aquaculture, of which most of it is cultured in pond systems. Asia supplies almost $90 \%$ of global aquaculture products, and aquaculture is a rapidly growing economic factor that promises high income gains but entails high environmental risks: mangrove deforestation, water pollution, and the use of antibiotics and pesticides increase the threats on coastal ecosystems [4].

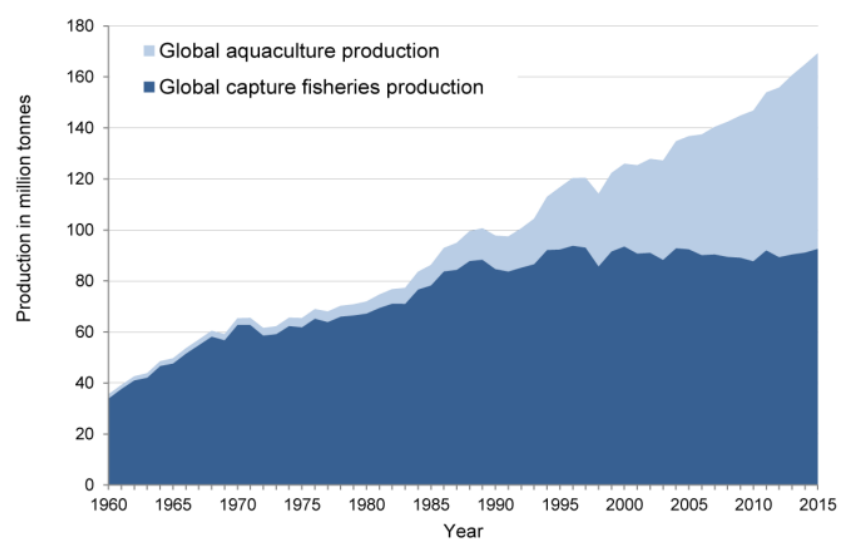

Figure 1: Global production of aquaculture and capture fisheries from 1960-2015. Data source: [1].

\section{POTENTIAL OF SAR FOR AQUACULTURE MAPPING}

Fish and shrimp are mainly produced in freshwater and brackish water pond systems in low topography areas along the coasts of South and East Asia. Ponds are permanently water-filled surfaces, surrounded by very narrow linear structures such as dams, dikes or levees with an average depth of less than 2 meters [4] and generally have a rectangular shape. Aquaculture pond sizes range from 
small-scale subsistence aquaculture farming (approx. 20x20m) to large commercial farms (several ha size).

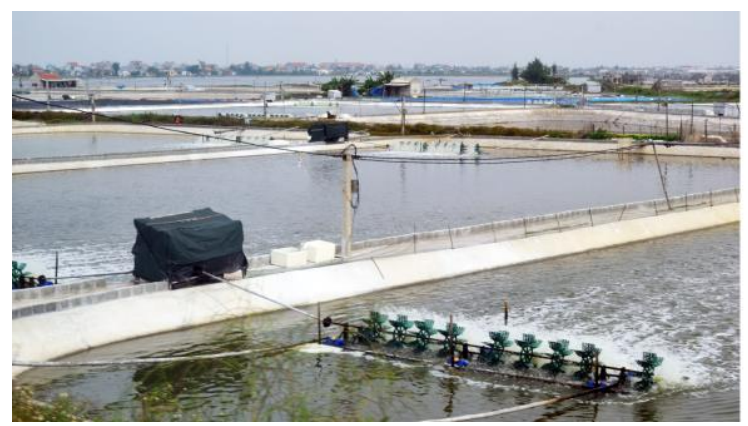

Figure 2: Intensive shrimp pond aquaculture in the Red River Delta, Vietnam.

With high resolution earth observation data we are able to detect aquaculture at single pond level. The great advantage of active microwave instruments for aquaculture mapping is their all-weather, day and night imaging capability which is particularly suited for cloud-prone coastal areas. The different backscatter response of the pond components (dikes and enclosed water surface; see figure 3) and its distinct rectangular structure allow for the separation of aquaculture areas from other surfaces. With dense time series data we are able to distinguish permanent water covered aquaculture ponds from other periodically water filled surfaces, such as paddy rice fields or flooded areas [5].

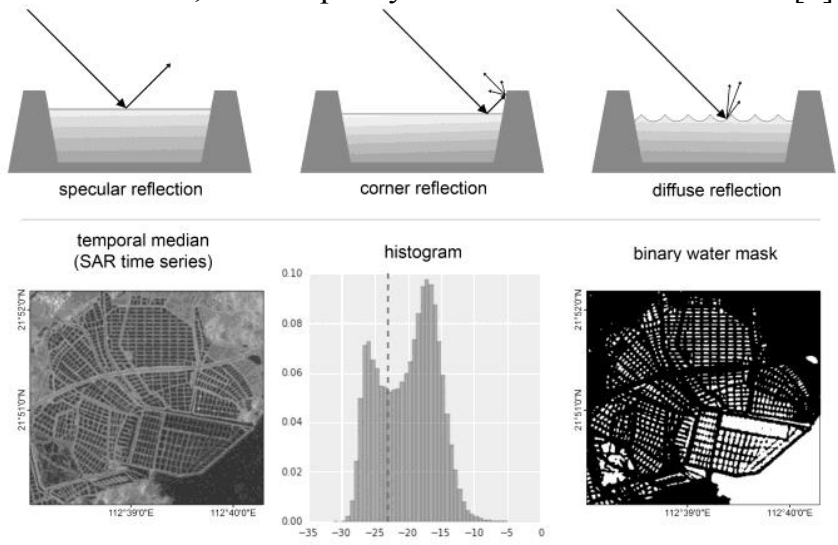

Figure 3: Radar interaction with ponds: specular, diffuse and corner reflection. Modified according to [5].

\section{DATA AND METHODOLOGY}

\subsection{SAR and terrain data}

High spatial resolution time series of free and open data acquired by the European Sentinel-1 radar satellites were collected and pre-processed. We used more than 1500 dualpolarized (VV/VH) Sentinel-1 scenes in interferometric wide swath (IW) mode and high resolution ground resolution detected (GRDH) format for coastal aquaculture hotspots along the Chinese and Vietnamese shoreline, e.g. the Pearl River Delta. To exclude water bodies, which are most likely to be confused with aquaculture ponds, we generated two terrain masks: For terrain masking we derived topographic information from SRTM Version 3.0 Global 1 arc second dataset and extracted elevation and slope thresholds to identify potential aquaculture areas. A detailed coastline dataset for Asia was provided by the Institute of Geographic Sciences and Natural Resources Research (IGSNRR), Chinese Academy of Sciences (CAS) and used to generate a binary land and water mask.

\subsection{Pre-processing}

The preprocessing comprised the application of an orbit file using restituted orbits, removal of thermal noise, and radiometric calibration to convert intensity values to the backscatter coefficient sigma naught. These pre-processing steps have been performed by the Google Earth Engine team [6]. For the study sites we used scenes in $\mathrm{VH}$ polarization and only from an ascending or descending orbit to reduce effects of orbit direction and look angle. Since the availability of scenes in ascending and descending orbits varies greatly from area to area we chose only the orbit direction which is more available for the specific study site.

\subsection{Thresholding and segmentation}

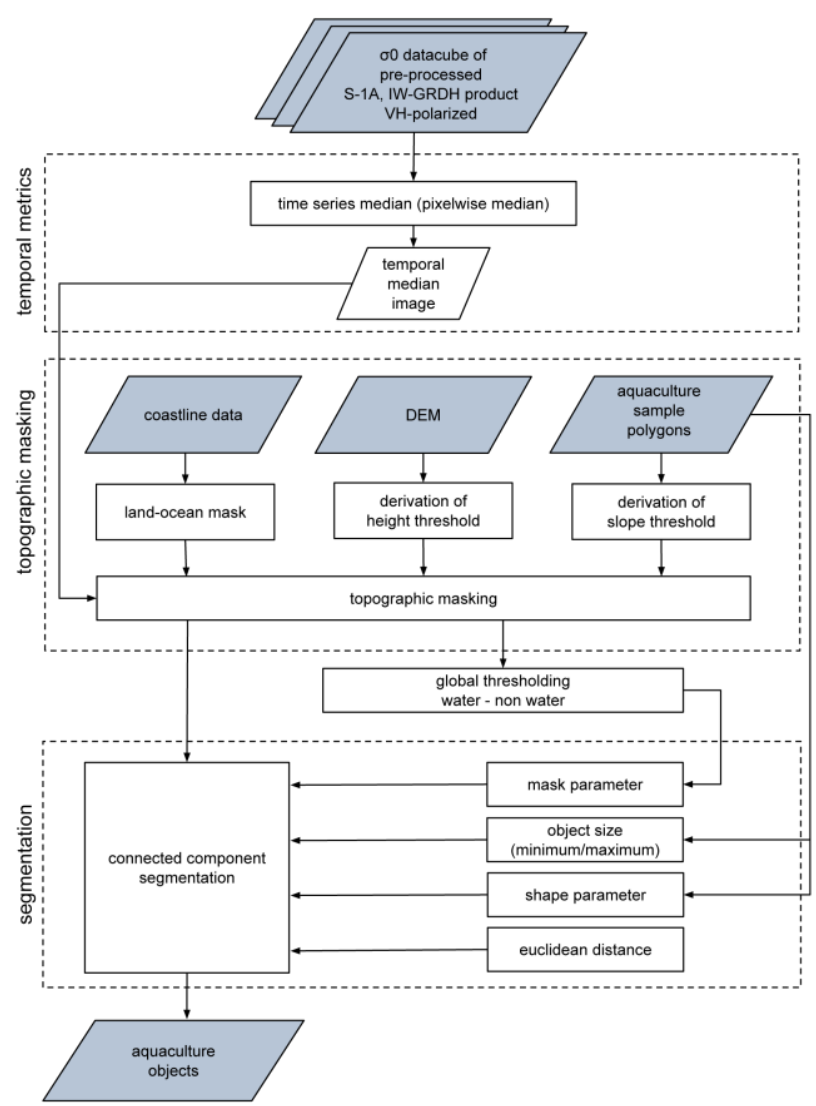

Figure 4: Flowchart. 
The pixel-wise median was calculated for the pre-processed time series data cube to reduce speckle noise in the intensity SAR imagery and identify permanent and stable low scatterers (see figure 4). By averaging over time, the median of the temporal data cube effectively improved the recognition and detection of small and narrow surface structures such as dams and levees surrounding aquaculture ponds. An automatic clustering-based image thresholding was used to mask land and water surfaces prior to the segmentation. We chose the OTSU method [7] to separate pond water and surrounding land area (dikes, levees, and dams). A connected component segmentation algorithm [8] was applied to the temporally filtered SAR time series data to extract pond objects based on shape and size features with a mean overall accuracy of 0.83 . Appropriate parameters for object area, elongation, region ratio were derived from an aquaculture pond sample dataset and applied for the object based image filtering.

\section{RESULTS AND DISCUSSION}
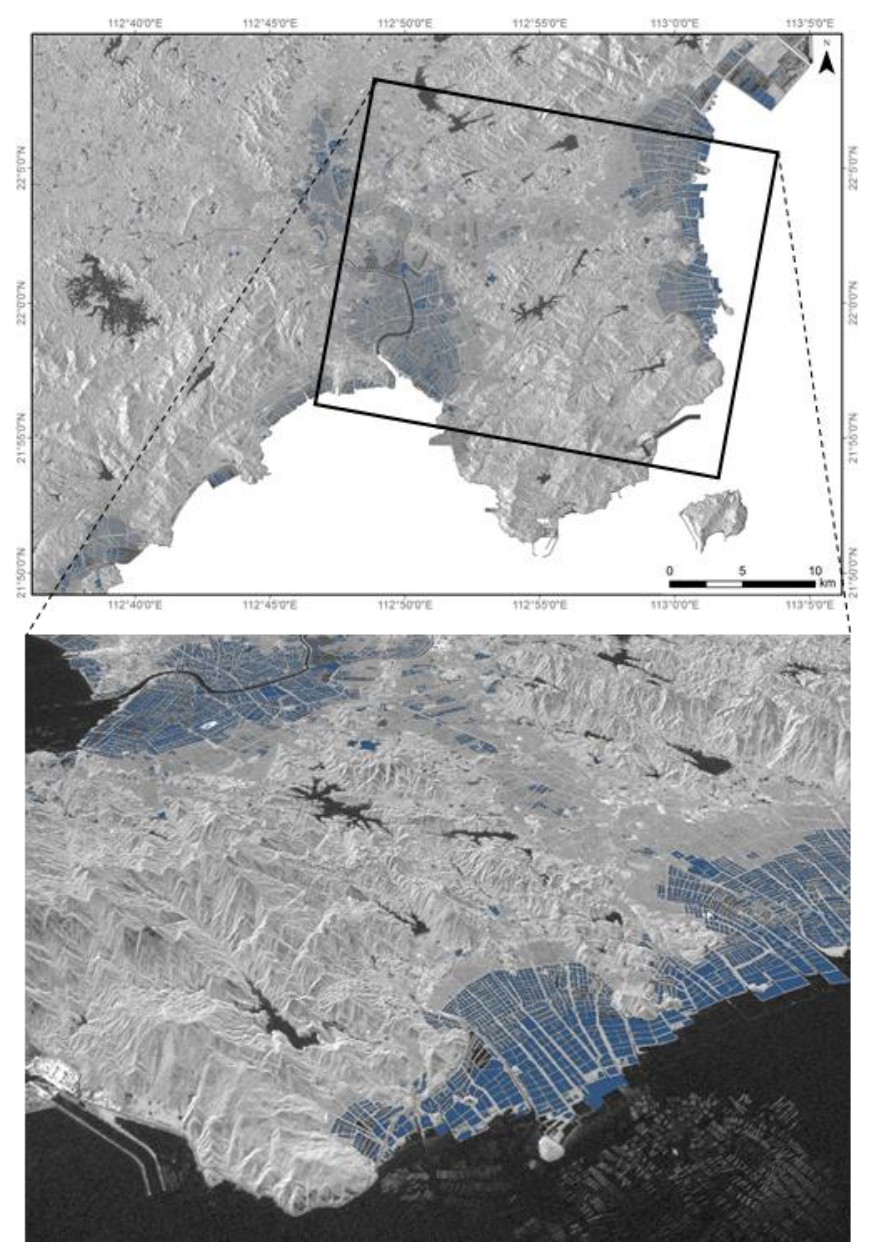

Figure 5: Earth observation derived coastal aquaculture ponds in the Pearl River Delta, Guangdong Province, China.
Coastal hotspot areas on the Chinese and Vietnamese coasts were investigated and the vast pond area required for the fish and shrimp farming becomes visible. As an example, figure 5 illustrates the results of the aquaculture mapping in the Pearl River Delta (China). Large-scale land use changes as well as loss and degradation of valuable wetlands are the consequence of the rapid aquaculture development. Considering increasing intensification in the aquaculture sector with higher use of pesticides and antibiotics [4], the protection of coastal ecosystems and their resources becomes a global challenge.

In view of the great potential of aquaculture to contribute to global food security, another research objective is the estimation of pond production quantities. Detailed data on aquaculture regional statistics, its expansion and localization barely exist but is of utmost interest as aquaculture is a major policy priority across governmental and nongovernmental institutions for the sustainable management [4] of our planet's coastal resources and to secure sufficient and nutritional food supplies for the growing world population. Although there exist national statistics (e.g. provided by the FAO for most countries) and also lower administrative units at sub-national levels, these data have generally limited detail and only provide statistics on total aquaculture quantities.

We collected annual statistics of aquaculture production for exemplary study sites on sub-provincial level and in-situ data on average harvest yields on pond level to estimate aquaculture production amounts for the earth observation derived fish and shrimp ponds. Such detailed production data and spatial information did not exist so far. With the SAR derived product it is possible for the first time to assess aquaculture on single pond level at regional scale and use that information for spatial analyses and production estimation.

\section{CONCLUSION}

The aim of this study was to assess aquaculture ponds from high resolution SAR data for coastal hotspots in Asia. The results proof that active microwave sensors with weather independent, day-and-night data-acquisition capabilities have great potential for pond detection and mapping on regional and global scale in cloud-prone coastal areas. The outcomes of this framework will be exploited for further global application and derivation of aquaculture ponds and to analyze the potential for the estimation of production quantities. Based on the presented results, earth observation can effectively support the sustainable development in the coastal zone and help to derive production volumes specifically for pond farms - the predominant culture system in the coastal zone of Asia. 


\section{REFERENCES}

[1] FAO. (2017). Fishery and Aquaculture Statistics. Global aquaculture production 1950-2015 (FishstatJ). In: FAO Fisheries and Aquaculture Department [online]. Rome. Updated 2017. Retrieved from

http://www.fao.org/fishery/statistics/software/fishstatj/en [2] S. H. Thilsted, A. Thorne-Lyman, P. Webb, J. R. Bogard, R. Subasinghe, M. J. Phillips, and E. H. Allison, "Sustaining healthy diets: The role of capture fisheries and aquaculture for improving nutrition in the post-2015 era", Food Policy, 61, pp. 126-131, 2016. https://doi.org/10.1016/j.foodpol.2016.02.005.

[3] HLPE. (2014). Sustainable fisheries and aquaculture for food security and nutrition. A report by the High Level Panel of Experts on Food Security and Nutrition of the Committee on World Food Security. Rome.

[4] M. Ottinger, K. Clauss, and C. Kuenzer, „Aquaculture: Relevance, distribution, impacts and spatial assessments - A review", Ocean and Coastal Management, 119, pp. 244-266, 2017. https://doi.org/10.1016/j.ocecoaman.2015.10.015.
[5] M. Ottinger, K. Clauss, and C. Kuenzer, „Large-Scale Assessment of Coastal Aquaculture Ponds with Sentinel-1 Time Series Data", Remote Sensing, 9(5), 440, 2017. https://doi.org/10.3390/rs9050440.

[6] N. Gorelick, M. Hancher, M. Dixon, S. Ilyushchenko, D. Thau, R. Moore, "Google Earth Engine: Planetary-scale geospatial analysis for everyone", Remote Sensing of Environment, 202, pp. 18-27. doi:10.1016/j.rse.2017.06.031

[7] N. Otsu, "A Threshold Selection Method from Gray-Level Histograms", IEEE Transactions on Systems, Man, and Cybernetics, 9(1), pp. 62-66, 1979.

[8] ORFEO ToolBox Development Team. OTB CookBook Documentation. Available online: https://www.orfeotoolbox.org/packages/OTBCookBook.pdf (accessed on 1 May 2017). 